\title{
Correction to: Enhanced Osteogenic Behavior of ADSCs Produced by Deproteinized Antler Cancellous Bone and Evidence for Involvement of ERK Signaling Pathway, by Wei J, Xu M, Zhang X, Meng S, Wang Y, Zhou T, Ma $Q$, Han B, Wei $Y$, and Deng $X$. Tissue Eng Part A 2015;21(11-12):1810-1821. DOI: 10.1089/ten.tea.2014.0395
}

\footnotetext{
TN ThE June 2015 issue of Tissue Engineering, Part A (vol. 21, no. 11-12, pages 1810-1821), in the article entitled Enhanced Osteogenic Behavior of ADSCs Produced by Deproteinized Antler Cancellous Bone and Evidence for Involvement of ERK Signaling Pathway, by Jinqi Wei, Mingming Xu, et al., the authors have discovered an error in the permission statement for Fig. 6A.
}

The corrected legend is reprinted here in full, with the corrected wording in bold:

FIG. 6. (A) Representative 3D $\mu$-CT images of rabbit mandibular bone defects in untreated group, CACB group, and CACB/ adipose-derived stem cells (ADSCs) group. Note that the image of the untreated group is adapted from Fig. $5 \mathrm{C}$ of our previous study ${ }^{\mathbf{1 6}}$ by permission of Elsevier Ltd. (B) Quantitative analysis of bone coverage, bone density, and bone volume at 12 weeks postimplantation. Scale bar $=8 \mathrm{~mm}$. Yellow circles denote the surgical site. $* p<0.05$ and $* * p<0.01$. Color images available online at www.liebertpub.com/tea.

The authors sincerely regret this error. 